Pacific Journal of Mathematics

STARLIKE AND CONVEX MAPS IN BANACH SPACES 


\section{STARLIKE AND CONVEX MAPS IN BANACH SPACES}

\section{T. J. SUFFrIDGE}

Let $X$ and $Y$ be complex Banach spaces and let $B=\{x \in$ $X:\|x\|<1\}$. This paper concerns holomorphic maps $f: B \rightarrow Y$ which have local holomorphic inverses. That is, for each $x \in B$, there is a neighborhood $N \subset Y$ of $f(x)$ and a holomorphic function $g: N \rightarrow B$ such that $g(f(x))=x$ and $f(g(y))=y$ for all $y \in N$. Necessary and sufficient conditions are found which guarantee that such a map be one-to-one and map the unit ball $B$ onto a domain which is convex or starlike with respect to 0 .

1. Introduction. When $X=Y=C$ (the complex plane), it is well known that if $f$ is holomorphic in $|z|<1$ and satisfies $f(0)=$ $0, f^{\prime}(0) \neq 0$ then $f$ is univalent and maps the disk onto a domain which is starlike with respect to 0 if and only if $\operatorname{Re}\left[z f^{\prime}(z) / f(z)\right]>0$ when $|z|<1$. Intuitively, this results from the fact that if we choose $z=r e^{i \theta}, 0<r<1, f(z) \neq 0$ and let $\phi(\theta)$ be a continuous branch of arg $f\left(r e^{i \theta}\right)$ locally then $\partial \phi / \partial \theta=\operatorname{Re}\left[z f^{\prime}(z) / f(z)\right]$. This idea does not extend readily to Banach spaces so we consider a different approach suggested by the following theorems due to M. S. Robertson [4].

THEOREM A. Let $w(z, t)=\sum_{1}^{\infty} b_{n}(t) z^{n}$ be regular in $|z|<1$ for $0 \leqq t \leqq 1$. Let $|w(z, t)|<1$ for $|z|<1,0 \leqq t \leqq 1, w(z, 0) \equiv z$. Let $\rho$ be a positive real number for which $w(z)=\lim _{t \rightarrow 0^{+}}(w(z, t)-z) / z t^{\rho}$ exists. Then $\operatorname{Re} w(z) \leqq 0$ for $|z|<1$. If $w(z)$ is also analytic in $|z|<$ 1 and $\operatorname{Re}(w(0)) \neq 0$, then $\operatorname{Re}(w(z))<0$ for $|z|<1$.

THEOREM B. Let $f(z)=z+a_{2} z^{2}+\cdots$ be regular and univalent in $|z|<1$. For $0 \leqq t \leqq 1$ let $F(z, t)$ be regular in $|z|<1$. Let $F(z$, $0) \equiv f(z)$ and $F(0, t) \equiv 0$. Let $\rho$ be a positive real number for which $F(z)=\lim _{t \rightarrow 0^{+}}(F(z, t)-F(z, 0)) / z t^{\circ}$ exists. Let $F(z, t)$ be subordinate to $f(z)$ in $|z|<1$ for $0 \leqq t \leqq 1$. Then $\operatorname{Re}\left(F(z) / f^{\prime}(z)\right) \leqq 0,|z|<1$. If in addition $F(z)$ is also analytic in $|z|<1$ and $\operatorname{Re}(F(0)) \neq 0$, then $\operatorname{Re}\left(f^{\prime}(z) / F(z)\right)<0,|z|<1$.

As observed by Robertson, if $f$ is holomorphic and univalent in $|z|<1, f(0)=0$, and $f(|z|<1)$ is starlike with respect to 0 then $(1-t) f(z)$ is subordinate to $f(z)$ (i.e., $(1-t) f(|z|<1) \subset f(|z|<1)$ ) for each $t, 0 \leqq t \leqq 1$. We may set $F(z, t)=(1-t) f(z)$ in Theorem B and obtain $F(z)=-f(z) / z$ when $\rho=1$. Thus we obtain the necessary 
condition $\operatorname{Re}\left[z f^{\prime}(z) / f(z)\right]>0$ by using the concept of subordination.

Now assume $f$ is holomorphic in $|z|<1, f(0)=0, f^{\prime}(z) \neq 0$ in $|z|<1$ and $w(z)=f(z) / z f^{\prime}(z)$ where $\operatorname{Re}(w(z))>0$ (equivalently $\operatorname{Re}\left[z f^{\prime}(z) / f(z)\right]>$ $0)$. Expanding $f^{-1}((1-t) f(z))$ in powers of $t$ about $t=0$, we have

$$
f^{-1}\left((1-t f(z))=z-t f(z) / f^{\prime}(z)+o(t)=z(1-t w(z))+o(t)\right.
$$

so $\left|f^{-1}((1-t) f(z))\right|<|z|$ when $0<t<t_{0}$ for some $t_{0}>0$. As we shall see later, this will imply $f$ is one-to-one and $f(|z|<1)$ is starlike with respect to 0 . This is the approach which is needed for extension of these ideas to Banach spaces.

It is well known that the image of the unit disk under a holomorphic map $f$ satisfying $f^{\prime}(0) \neq 0$ is convex if and only if the function $z f^{\prime}(z)$ has an image which is starlike with respect to 0 . The generalization of this result to Banach spaces is false. In fact, we will show that if $X=\ell^{1}$ then $f: B \rightarrow Y$ is a biholomorphic map of $B$ onto a convex domain if and only if $f$ is a bounded linear map having a bounded inverse (Corollary 1). We will also show (Corollary 2) that if $X=$ $\ell^{\infty}$ and $f: B \rightarrow Y$ is a biholomorphic map of $B$ onto a convex domain then $f=L \circ g$ where $g: B \rightarrow X$ is given by $g=\left(g_{1}, g_{2}, \cdots\right), g_{k}(x)=$ $g_{k}\left(x_{k}\right)=x_{k}+a_{2 k} x_{k}^{2}+\cdots, x=\left(x_{1}, x_{2}, \cdots\right)$ and $L$ is a bounded linear map having a bounded inverse (i.e., $f$ is a linear map composed with a function whose coordinates are functions of one variable only).

See [6] for extension of Robertson's Theorems $\mathrm{A}$ and $\mathrm{B}$ to $C^{n}$ and see [3] and [6] for some results concerning starlikeness and convexity in $C^{n}$.

2. Starlike maps in Banach spaces, Let $B_{r}=\{x \in X:\|x\|<r\}$ and $B=B_{1}$. For $0 \neq x \in X$, let $T(x)$ be the collection of all continuous real linear functionals $x^{\prime}$ on $X$ (regarded as a real linear space) satisfying $x^{\prime}(x)=\|x\|$ and $x^{\prime}(y) \leqq\|y\|$ for all $y \in X$. By the Hahn-Banach theorem, $T(x)$ is nonempty. Also, if $x^{\prime} \in T(x)$ then $\left\{y \in X: x^{\prime}(y)=\|x\|\right\}$ is a supporting hyperplane for the convex set $B_{\|x\|^{\bullet}}$. Let $\mathscr{P}_{0}(B)$ be the class of mappings $w: B \rightarrow X$ which are holomorphic and satisfy $w(0)=$ 0 and $x^{\prime}(w(x)) \geqq 0$ when $0 \neq x \in B$ and $x^{\prime} \in T(x)$. Further, let $\mathscr{P}(B)$ be the class of $w \in \mathscr{P}_{0}(B)$ which satisfy $x^{\prime}(w(x))>0$ when $0 \neq x \in B$ and $x^{\prime} \in T(x)$.

ExAmples. If $X=C$ and $z_{0} \neq 0$ then $T\left(z_{0}\right)$ contains only the functional $x^{\prime}$ given by $x^{\prime}(z)=\operatorname{Re}\left[\left|z_{0}\right| z / z_{0}\right]$ and $\mathscr{P}(B)$ consists of the class of $w$ such that $w(z) / z$ is holomorphic in $|z|<1$ and $\operatorname{Re}[w(z) / z]>0$.

If $X=C^{n}$ with sup norm, and $0 \neq x \in X$ then $T(x)$ consists of those functionals $x^{\prime}$ given by $x^{\prime}(y)=\sum_{\mid x_{k i}=\|x\|} t_{k}\|x\| \operatorname{Re}\left(y_{k} / x_{k}\right)$ where $t_{k} \geqq 0$ for each $k$ and $\sum_{\left|x_{k}\right|=|x| \mid} t_{k}=1$. In this case, $\mathscr{P}(B)$ is the class 
of $w: B \rightarrow X$ which are holomorphic and satisfy $w(0)=0$ and $\operatorname{Re}\left[w_{j}(x) / x_{j}\right]>0$ when $\|x\|=\left|x_{j}\right|>0$. We remark that $\mathscr{P}_{0}(B)$ in this case is the class $\mathscr{P}$ defined in [6].

If $X=\ell^{p}, 1<p<\infty$, and $0 \neq x \in X$ then $T(x)$ contains only the functional $x^{\prime}$ given by $x^{\prime}(y)=\operatorname{Re}\left(\sum\left|x_{j}\right|^{p} y_{j} / x_{j}\right) /\|x\|^{p-1}$ and $\mathscr{P}(B)$ consists of those holomorphic $w: B \rightarrow \ell^{p}$ satisfying $w(0)=0$ and $\operatorname{Re}\left(\sum\left|x_{j}\right|^{p} w_{j}(x) / x_{j}\right)>0$.

If $X=\ell^{1}$ and $0 \neq x \in X$ then $T(x)$ consists of those functionals $x^{\prime}$ given by

$$
x^{\prime}(y)=\operatorname{Re}\left(\sum_{x_{j} \neq 0}\left|x_{j}\right| y_{j} \mid x_{j}+\sum_{x_{j}=0} \alpha_{j} y_{j}\right)
$$

where $\alpha_{j}$ satisfy $\left|\alpha_{j}\right| \leqq 1$. In this case, $\mathscr{P}(B)$ consists of those holomorphic $w: B \rightarrow \ell^{1}$ satisfying $w(0)=0$ and $\operatorname{Re}\left[\sum_{x_{j} \neq 0}\left|x_{j}\right| w_{j}(x) / x_{j}-\right.$ $\left.\sum_{x_{j}=0}\left|w_{j}(x)\right|\right]>0$. In the finite dimensional case using the $p$ norm, $1 \leqq p<\infty$ the class $\mathscr{P}_{0}(B)$ is the class $\mathscr{P}_{p}$ defined in [6].

The following lemmas generalize Robertson's Theorems A and B to Banach spaces. They also include Lemmas 1-4 of [6].

LEMMA 1. Let $v(x, t): B \times I \rightarrow B$ be holomorphic for each $t \in I=$ $[0,1], v(0, t)=0$ and $v(x, 0)=x . \quad$ If $\lim _{t \rightarrow 0^{+}}[(x-v(x, t)) / t]=w(x)$ exists and is holomorphic in $B$ then $w \in \mathscr{P}_{0}(B)$.

Proof. Let $0 \neq x \in B$ and $x^{\prime} \in T(x)$. By Schwarz lemma, $\|v(x, t)\| \leqq$ $\|x\|$ so $x^{\prime}[(x-v(x, t)) / t]=\left(\|x\|-x^{\prime}(v(x, t))\right) / t \geqq(\|x\|-\|v(x, t)\|) / t \geqq 0$ and the desired result follows by continuity of $x^{\prime}$.

The following example shows that there are nontrivial cases in which the limit function $w$ of Lemma 1 is not in the class $\mathscr{P}(B)$.

ExAmple. Let $X=C^{2}$ with $\|x\|^{2}=\left|x_{1}\right|^{2}+\left|x_{2}\right|^{2}$. For $0 \leqq t \leqq 1$, let $v(x, t): B \rightarrow B$ be the restriction of the linear map having matrix

$$
\left(\begin{array}{cc}
\sqrt{1-t^{2}} & t \\
-t & \sqrt{1-t^{2}}
\end{array}\right)
$$

Then the limit function $w$ of Lemma 1 is given by $w(x)=\left(-x_{2}, x_{1}\right)$. If $x^{\prime} \in T(x)$ then $x^{\prime}(y)=\operatorname{Re}\left(\bar{x}_{1} y_{1}+\bar{x}_{2} y_{2}\right) /\|x\|$ so $x^{\prime}(w(x))=\operatorname{Re}\left(-\bar{x}_{1} x_{2}+\right.$ $\left.x_{2} \bar{x}_{1}\right) /\|x\| \equiv 0$.

LEMma 2. Let $f: B \rightarrow Y$ be a biholomorphic map (holomorphic with a holomorphic inverse) of $B$ onto an open set $f(B) \subset Y$ and let $f(0)=0$. Let $F(x, t): B \times I \rightarrow Y$ be a holomorphic function of $x$ for each $t \in I, F(x, 0)=f(x), F(0, t)=0$ and suppose $F(B, t) \subset f(B)$ for each $t \in I$. Further, suppose $\lim _{t \rightarrow 0^{+}}[(F(x, 0)-F(x, t)) / t]=F(x)$ exists and is holomorphic. Then $F(x)=D f(x)(w(x))$ where $w \in \mathscr{P}_{0}(B)(D f(x)$ is the Fréchet derivative of $f$ at $x)$. 
Proof. Since $F(B, t) \subset f(B)$ for each $t \in I$, there exists $v: B \times I \rightarrow$ $B\left(v(x, t)=f^{-1}(F(x, t))\right)$ holomorphic for each $t \in I$ such that $f(v(x, t))=$ $F(x, t)$ where $\|v(x, t)\| \leqq\|x\|$. Fix $x,\|x\|>0$. Then

$$
f(v(x, t))=f(x)+D f(x)(v(x, t)-x)+R(v(x, t), x)
$$

where $\|R(y, x)\| /\|y-x\| \longrightarrow 0$ as $\|y-x\| \longrightarrow 0$. Therefore

$$
\frac{F(x, 0)-F(x, t)}{t}=D f(x)\left(\frac{x-v(x, t)}{t}-\frac{R(v(x, t), x)}{t}\right) .
$$

We wish to conclude $R(v(x, t), x) / t \rightarrow 0$ as $t \rightarrow 0^{+}$so that $\lim _{t \rightarrow 0^{+}}((x-$ $v(x, t)) / t$ ) exists and an application of Lemma 1 completes the proof. We first show that $\|x-v(x, t)\| / t$ is bounded as $t \rightarrow 0^{+}$. Suppose for some sequence $\left\{t_{n}\right\}, t_{n} \rightarrow 0^{+}$and $\left\|x-v\left(x, t_{n}\right)\right\| / t_{n} \rightarrow \infty$. We have

$$
F(x)=\lim _{n \rightarrow \infty}\left\{\left[D f(x)\left(\frac{x-v\left(x, t_{n}\right)}{\left\|x-v\left(x, t_{n}\right)\right\|}\right)-\frac{R\left(v\left(x, t_{n}\right), x\right)}{\left\|x-v\left(x, t_{n}\right)\right\|}\right] \frac{\left\|x-v\left(x, t_{n}\right)\right\|}{t_{n}}\right\}
$$

and since $R\left(v\left(x, t_{n}\right), x\right) /\left\|x-v\left(x, t_{n}\right)\right\| \rightarrow 0$ we must also have $D f(x)((x-$ $\left.\left.v\left(x, t_{n}\right)\right) /\left\|x-v\left(x, t_{n}\right)\right\|\right) \rightarrow 0$. This contradicts the assumption $f^{-1}$ is holomorphic so such a sequence cannot exist, i.e., $\|x-v(x, t)\| / t$ is bounded as $t \rightarrow 0^{+}$. The desired conclusion now clearly follows.

If $X=C$ and $w \in \mathscr{P}_{0}(B)$ then $\operatorname{Re}(w(z) / z) \geqq 0$ when $|z|<1$. It is then easy to see that for $|\alpha|<1, w(\alpha z) / \alpha \in \mathscr{P}_{0}(B)$ and in fact $w \in$ $\mathscr{P}(B)$ unless $\operatorname{Re} w^{\prime}(0)=0$ and in this case $w$ is constant. This result takes the following form in normed linear spaces.

Lemma 3. If $w \in \mathscr{P}_{0}(B)$ and $|\alpha|<1$ then $\frac{1}{\alpha} w(\alpha x) \in \mathscr{P}_{0}(B)\left(\frac{1}{\alpha} w(\alpha x)\right.$ is understood to be the limit value

$$
D w(0)(x) \text { when } \alpha=0) \text {. }
$$

Further, if $x^{\prime} \in T(x), 0<\|x\|<1$ then $x^{\prime}(w(x))=0$ if and only if $x^{\prime}(D w(0)(x))=0$ and in this case $x^{\prime}(1 / \alpha \cdot w(\alpha x)) \equiv 0$ when $|\alpha|<1 /\|x\|$.

Proof. For $0<|\alpha|<1$ and $x^{\prime} \in T(x)$, define $x_{\alpha}^{\prime}$ by $x_{\alpha}^{\prime}(y)=x^{\prime}(|\alpha| y / \alpha)$ for all $y \in X$. Then $x_{\alpha}^{\prime}(\alpha x)=\|\alpha x\|$ and $x_{\alpha}^{\prime}(y) \leqq\|y\|$ for all $y \in X$ so $x_{\alpha}^{\prime} \in T(\alpha x)$. Therefore,

$$
0 \leqq \frac{1}{|\alpha|} x_{\alpha}^{\prime}(w(\alpha x))=x^{\prime}(w(\alpha x) / \alpha)
$$

and by continuity of $x^{\prime}, x^{\prime}(D w(0)(x)) \geqq 0$. Thus we have shown $w(\alpha x) / \alpha \epsilon$ $\mathscr{P}_{0}(B)$ when $|\alpha|<1$.

Also, $x^{\prime}(y)=\operatorname{Re}\left[x^{\prime}(y)-i x^{\prime}(i y)\right]$ is the real part of a continuous 
complex linear functional so $x^{\prime}(w(\alpha x) / \alpha)$ is a nonnegative harmonic function of $\alpha$ for fixed $x, \mid \alpha,<1 /\|x\|$. Therefore $x^{\prime}(w(\alpha x) / \alpha)>0$ or $x^{\prime}(w(\alpha x) / \alpha) \equiv 0$ (x fixed). This completes the proof.

Definition 1. A holomorphic map $f: B \rightarrow Y$ is starlike if $f$ is one-to-one, $f(0)=0$ and $(1-t) f(B) \subset f(B)$ for all $t \in I$.

THEOREM 1. Suppose $f: B \rightarrow Y$ is starlike and that $f^{-1}$ is holomorphic on $f(B)$ open $\subset Y$. There exists $w \in \mathscr{P}(B)$ such that

$$
f(x)=D f(x)(w(x)) \text {. }
$$

Proof. Apply Lemma 2 with $F(x, t)=(1-t) f(x)$ to obtain (1) with $w \in \mathscr{P}_{0}(B)$. Since

$$
\frac{1}{\alpha} f(\alpha x)=D f(\alpha x)\left(\frac{1}{\alpha} w(\alpha x)\right)
$$

for $|\alpha|<1$, letting $\alpha \rightarrow 0$ we have

$$
D f(0)(x)=D f(0)(D w(0)(x))
$$

so $D w(0)(x)=x$ since $f^{-1}$ holomorphic implies $D f(0)$ is invertible. Using Lemma 3 we conclude $w \in \mathscr{P}(B)$.

We have the following "converse" to Theorem 1.

Theorem 2. Let $f: B \rightarrow Y$ be holomorphic and $f(0)=0$. Assume $D f(x)$ has a bounded inverse for each $x \in B$ (hence $f^{-1}$ exists and is holomorphic in a neighborhood of each point of $f(B)$ ) and that for some $w \in \mathscr{P}(B), f(x)=D f(x)(w(x))$. Suppose further that for each $r$, $0<r<1$, there exists $M(r)$ such that $\left\|[D f(x)]^{-1}\right\| \leqq M(r)$ when $\|x\| \leqq$ $r$. Then $f$ is starlike.

Proof. We first observe that $f(x) \neq 0$ if $x \neq 0$ for $f(x)=0$ implies $D f(x)(w(x))=0$ so $w(x)=0$ since $D f(x)$ is invertible. But $x^{\prime} \in T(x)$ implies $x^{\prime}(w(x))>0$ so $w(x) \neq 0$ when $x \neq 0$.

For $0 \neq x \in B$, let $N_{x}$ be a neighborhood of $f(x)$ in which $f^{-1}$ exists as a holomorphic function. Let $v(x, t)=f^{-1}((1-t) f(x))$ for $-t_{0}<t<t_{1}$ where $t_{0}$ and $t_{1}$ are positive such that $(1-t) f(x) \in N_{x}$ when $-t_{0}<t<t_{1}$. We wish to show $\|v(x, t)\|$ is strictly decreasing as a function of $t$. Note that

$$
\begin{aligned}
v(x, t) & =v(x, 0)+[D f(x)]^{-1}(-t f(x))+o(t) \\
& =x-t w(x)+o(t)
\end{aligned}
$$

so for $x^{\prime} \in T(x)$ we have 


$$
\begin{aligned}
\|v(x, t)\| & \geqq x^{\prime}(v(x, t))=x^{\prime}(x)-t x^{\prime}(w(x))+o(t) \\
& =\|x\|-t x^{\prime}(w(x))+o(t)>\|x\|
\end{aligned}
$$

when $t$ is negative, $|t|$ sufficiently small. But we may apply this result at $y=v(x, t)$ (so $v(y, \tau)=f^{-1}((1-\tau)(1-t) f(x))$ ) to conclude that $\|v(x, t)\|$ is strictly decreasing in $-t_{0}<t<t_{1}$.

For $0 \leqq t \leqq 1$, let $A_{t}=\{(1-\tau) f(x): 0 \leqq \tau \leqq t\}$ and let $T=\{t \epsilon$ [0,1]: there exists a neighborhood $N_{t}$ of $A_{t}$ such that $f^{-1}$ exists as a holomorphic function on $N_{t}$ such that $f^{-1}(f(x))=x$. It is easy to see that $T$ is open and nonempty. We wish to show that $T$ is closed so $T=[0,1]$. Let $0<t_{2} \leqq 1$ where $0 \leqq t<t_{2}$ implies $t \in T$. We wish to show $t_{2} \in T$. Define

$$
L_{\tau}=\int_{0}^{\tau}\left\|\left[D f\left(f^{-1}((1-t) f(x))\right)\right]^{-1}(-f(x))\right\| d t .
$$

Computing the derivative of $f^{-1}((1-t) f(x))$ with respect to $t$, we see that the integral (2) gives the length of the arc $f^{-1}\{(1-t) f(x)$ : $0 \leqq t \leqq \tau\}$. Note also that for $0 \leqq \tau<t_{0},\left\|f^{-1}((1-t) f(x))\right\| \leqq\|x\|$ in the integrand since $\left\|f^{-1}((1-t) f(x))\right\|$ decreases with $t$. Hence by hypothesis, the integrand is continuous and bounded by $M(\|x\|)\|f(x)\|$ so the integral exists for $0 \leqq \tau \leqq t_{2}$. Further, if $0 \leqq \tau_{1}<\tau_{2}<t_{2}$ we have

$$
\begin{aligned}
L_{\tau_{2}}-L_{\tau_{1}} & =\int_{\tau_{1}}^{\tau_{2}}\left\|\left[D f\left(f^{-1}((1-t) f(x))\right)\right]^{-1}(-f(x))\right\| d t \\
& \geqq\left\|f^{-1}\left(\left(1-\tau_{2}\right) f(x)\right)-f^{-1}\left(\left(1-\tau_{1}\right) f(x)\right)\right\| .
\end{aligned}
$$

Let $\left\{\tau_{k}\right\}_{k=1}^{\infty}$ be an increasing sequence of values of $\tau$ such that $L_{\tau_{k}}=$ $\left(1-2^{-k}\right) L_{t_{2}}$. Then

$$
\begin{aligned}
L_{t_{2}}\left|2^{-m}-2^{-n}\right| & =\left|L_{\tau_{m}}-L_{\tau_{n}}\right| \\
& \geqq\left\|f^{-1}\left(\left(1-\tau_{m}\right) f(x)\right)-f^{-1}\left(\left(1-\tau_{n}\right) f(x)\right)\right\|
\end{aligned}
$$

so $\left\{f^{-1}\left(\left(1-\tau_{k}\right) f(x)\right)\right\}_{k=1}^{\infty}$ is a Cauchy sequence which converges to some value $y \in X$ such that $\|y\| \leqq\|x\|$. Clearly $f(y)=\left(1-t_{2}\right) f(x)$. Let $G$ be a neighborhood of $f(y)$ such that $f^{-1}$ exists uniquely in $G$ as a holomorphic function and $f^{-1}(f(y))=y$. Since some of the values $(1-$ t) $f(x)$ lie in $G$ for $t<t_{2}$, the values of $f^{-1}$ in $G$ and in $N_{t}$ must agree for these values of $t$. Therefore, $\left(\bigcup_{0 \leqq t<t_{2}} N_{t}\right) \cup G$ is a neighborhood of $A_{t_{2}}$ on which $f$ has a unique holomorphic inverse. Thus we have proved $t_{2} \in T$ and $T$ is closed.

Now suppose $f(x)=f(y), x \neq y$. Then there exist inverses $g_{1}$ and $g_{2}$ of $f$ holomorphic in a connected neighborhood $N$ of $A=\{(1-$ t) $f(x): 0 \leqq t \leqq 1\}$ such that $g_{1}(f(x))=x$ and $g_{2}(f(x))=y$. Clearly, the set of points on which $g_{1}$ and $g_{2}$ agree is both open and closed (relative to $N$ ) so either $g_{1} \equiv g_{2}$ or $g_{1}(u)$ is never equal to $g_{2}(u), u \in N$. 
But $0 \in N$ and $g_{1}(0)=g_{2}(0)=0$ while also $f(x) \in N$ and $g_{1}(f(x))=x \neq$ $y=g_{2}(f(x))$. This contradiction completes the proof of the theorem.

REMARK. We do not know whether Theorem 2 remains true without assuming $X$ is complete or without the boundedness condition on $[D f(x)]^{-1}$. The usefulness of this boundedness condition comes from the fact that for $r<1$ this condition implies that the boundary of $f\left(B_{r}\right)$ (as a subset of $Y$ rather than $\left.f(B)\right)$ is $f(\|x\|=r$ ).

Example. Let $X=Y=\ell^{\infty}$ and define $f: B \rightarrow Y$ by $f(x)=\left(f_{1}\right.$, $\left.f_{2}, \cdots\right)$ where $f_{1}(x)=x_{1}$ and $f_{j}(x)=x_{j}\left(1-x_{1}\right)$ if $j \geqq 2$. Then

$$
[D f(x)]^{-1}(f(x))=w(x)=\left(w_{1}, w_{2}, \cdots\right)
$$

where $w_{1}=x_{1}$ and $w_{j}=x_{j} /\left(1-x_{1}\right)$ if $j \geqq 2$. Suppose $0 \neq x \in B$ and $x^{\prime} \in T(x)$. If $\left|x_{1}\right|<\|x\|$ then $x^{\prime}(1,0,0, \cdots)=0=x^{\prime}(i, 0,0, \cdots)$ for otherwise we may choose $\alpha$ so that $\|x\|=\|x+(\alpha, 0,0, \cdots)\|$ and $\|x\|<x^{\prime}(x)+x^{\prime}(\alpha, 0,0, \cdots)=x^{\prime}(x+(\alpha, 0,0, \cdots))$.

If $\|x\|=\left|x_{1}\right|$, then $x^{\prime}\left(x_{1}, 0,0, \cdots\right) \geqq 0$ otherwise, choose $\alpha=-$ $t x_{1}$ where $t$ is small and positive so that $\|x+(\alpha, 0,0, \cdots)\| \leqq\|x\|$ and $x^{\prime}(x+(\alpha, 0,0, \cdots))>x^{\prime}(x)=\|x\|$.

Also, if $\|x\|=\left|x_{1}\right|$ then $x^{\prime}\left(i x_{1}, 0,0, \cdots\right)=0$ since $\sqrt{1+t^{2}}\|x\| \geqq$ $x^{\prime}\left((1 \pm i t) x_{1}, x_{2}, \cdots\right)=x^{\prime}(x) \pm t x^{\prime}\left(i x_{1}, 0,0, \cdots\right)$ so

$$
\sqrt{\frac{1+t^{2}}{|t|}}\|x\| \geqq \pm x^{\prime}\left(i x_{1}, 0,0, \cdots\right) \text {. }
$$

Similarly, $x^{\prime}\left(i\left(0, x_{2}, x_{3}, \cdots\right)\right)=0$ and $x^{\prime}\left(0, x_{2}, x_{3}, x_{4}, \cdots\right) \geqq 0$. Therefore

$$
\begin{aligned}
x^{\prime}(w(x)) & =x^{\prime}\left(x_{1}, 0,0, \cdots\right)+x^{\prime}\left(\frac{1}{1-x_{1}}\left(0, x_{2}, x_{3}, \cdots\right)\right) \\
& =x^{\prime}\left(x_{1}, 0,0, \cdots\right)+\operatorname{Re}\left(\frac{1}{1-x_{1}} x^{\prime}\left(0, x_{2}, x_{3}, \cdots\right)\right) \\
& >0
\end{aligned}
$$

so $f$ is starlike. Note that as $x$ tends to a boundary point of the form $\left(1, x_{2}, x_{3}, \cdots\right), f(x)$ tends to the point $(1,0,0,0, \cdots)$.

3. Convex maps of the unit ball. We wish to obtain necessary and sufficient conditions that $f: B \rightarrow Y$ be a biholomorphic map of $B$ onto a convex domain in $Y$. We begin with the following lemma.

LEMMA 4. If $f: B \rightarrow Y$ is a biholomorphic map of $B$ onto a convex domain then $f\left(B_{r}\right)$ is convex for each $r, 0 \leqq r \leqq 1$. 
Proof. Suppose $x_{0}, y \in B,\|y\| \leqq\left\|x_{0}\right\|$ and $x_{0} \neq 0$. Then $t f\left(x_{0}\right)+$ $(1-t) f(y) \in f(B)$ when $0 \leqq t \leqq 1$ and we wish to show $t f\left(x_{0}\right)+$ $(1-t) f(y) \in f\left(B_{r}\right)$ when $\left\|x_{0}\right\|<r$. Let $\ell$ be a (complex) continuous linear functional satisfying $\ell\left(x_{0}\right)=1,\|\ell\| \leqq 1 /\left\|x_{0}\right\|$. Let $t f(x)+(1-$ t) $f(\ell(x) y)=f(v(x, t))$. Then $v(0, t)=0$ and $\|v(x, t)\|<1$ so $\|v(x, t)\| \leqq$ $\|x\|$. Hence $f\left(v\left(x_{0}, t\right)\right)=t f\left(x_{0}\right)+(1-t) f(y)$ where $\left\|v\left(x_{0}, t\right)\right\| \leqq\left\|x_{0}\right\|$ and the lemma is proved.

Definition 2. If $f: B \rightarrow Y$ is a biholomorphic map of $B$ onto a convex domain, we say that $f$ is convex.

If $X=C$, it is well known than a function $f$ holomorphic in $|z|<$ 1 satisfying $f^{\prime}(0) \neq 0$ is convex if and only if $\operatorname{Re}\left[z f^{\prime \prime}(z) / f^{\prime}(z)+1\right]>0$ in $|z|<1$. The analogous condition for a general Banach space is necessary but not sufficient.

Theorem 3. If $f: B \rightarrow Y$ is convex then

$$
D^{2} f(x)(x, x)+D f(x)(x)=D f(x)(w(x))
$$

where $w \in \mathscr{P}(B)$.

Proof. Let $F(x, t)=1 / 2\left(f\left(e^{i \sqrt{t}} x\right)+f\left(e^{-i \sqrt{t}} x\right)\right)$ for $t \in I$. Then $F(x$, $t$ ) satisfies the hypotheses of Lemma 2 with

$$
\begin{aligned}
F(x)= & \lim _{t \rightarrow 0^{+}} \frac{f(x)-\frac{1}{2}\left(f\left(e^{i \sqrt{t}} x\right)+f\left(e^{-i \sqrt{t}} x\right)\right)}{t} \\
= & \lim _{t \rightarrow 0^{+}}-\frac{1}{2 t}(D f(x)((2 \cos \sqrt{t}-2) x) \\
& \left.+D^{2} f(x)(i \sin \sqrt{t} x, i \sin \sqrt{t} x)+o(t)\right) \\
= & \frac{1}{2}\left(D f(x)(x)+D^{2} f(x)(x, x)\right) .
\end{aligned}
$$

Hence (3) follows with $w \in \mathscr{P}_{0}(B)$. Again, $D w(o)(x)=x$ and by Lemma $3, w \in \mathscr{P}(B)$.

The example $X=C^{2}$ with sup norm and $f\left(z_{1}, z_{2}\right)=\left(z_{1}+z_{2}^{2} / 2, z_{2}\right)$ shows the condition is not sufficient ( $f$ is not convex by Corollary 2 below).

Let $Q_{0}(B)$ be the class of functions $w: B \times B \rightarrow X$ which are holomorphic in each variable and which satisfy $w(x, x)=0$ and $x^{\prime}(w(x$, $y)) \geqq 0$ when $x^{\prime} \in T(x)$ and $\|y\| \leqq\|x\|$. Let $Q(B)$ be the collection of all $w \in Q_{0}(B)$ which satisfy $x^{\prime}(w(x, y))>0$ when $x^{\prime} \in T(x)$ and $\|y\|<$ $\|x\|$.

Lemma 5. If $w \in Q_{0}(B)$ and $|\alpha|<1$ then $1 / \alpha \cdot w(\alpha x, \alpha y) \in Q_{0}(B)$ (the 
limit value at $\alpha=0$ is $D w(0,0)(x, y))$. Further, if $x^{\prime} \in T(x), 0 \neq x \in B$, and $\|y\| \leqq\|x\|$ then $x^{\prime}(w(x, y))=0$ if and only if $x^{\prime}(D w(0,0)(x, y))=0$.

Proof. See the proof of Lemma 3.

Theorem 4. Suppose $f: B \rightarrow Y$ is convex. Then $f(x)-f(y)=$ $D f(x)(w(x, y))$ where $w \in Q(B)$.

Proof. Since $(1-t) f(x)+t f(y) \in f(B)$ for all $x, y \in B$ when $0 \leqq$ $t \leqq 1$, there exists $v(x, y, t): B \times B \times I \rightarrow B$ such that $f(v(x, y, t))=$ $(1-t) f(x)+t f(y)$. By Lemma $4,\|v(x, y, t)\| \leqq \max (\|x\|,\|y\|)$. Also,

$$
f(v(x, y, t))=f(x)+D f(x)(v(x, y, t)-x)+R(v(x, y, t), x)
$$

so

$$
\begin{aligned}
f(x)-f(y) & =\lim _{t \rightarrow 0^{+}} \frac{f(x)-f(v(x, y, t))}{t} \\
& =\lim _{t \rightarrow 0^{+}}\left(D f(x)\left(\frac{x-v(x, y, t)}{t}\right)-\frac{1}{t} g(v(x, y, t), x)\right) \\
& =D f(x)(w(x, y)) .
\end{aligned}
$$

Since $w(x, y)=\lim _{t \rightarrow 0^{+}}(x-v(x, y, t)) / t$, it is clear that $w \in Q_{0}(B)$. Since $D w(0,0)(x, y)=x-y$, Lemma 5 implies that $w \in Q(B)$.

Again assuming a boundedness condition on $[D f(x)]^{-1}$ we can prove the converse.

Theorem 5. Suppose $f: B \rightarrow Y$ is holomorphic, $D f(x)$ has bounded inverse for each $x \in B$ and that for some $w \in Q(B), f(x)-f(y)=$ $D f(x)(w(x, y))$. Suppose further that for each $r, 0<r<1$, there exists $M(r)>0$ such that $\left\|[D f(x)]^{-1}\right\| \leqq M(r)$ when $\|x\| \leqq r$. Then $f$ is convex.

Proof. Since $w(x, 0) \in \mathscr{P}(B), f(x)-f(0)$ is starlike. Let $v(x, y, t)=f^{-1}((1-t) f(x)+t f(y))$ when $x, y \in B$ and $t \in\left[0, t_{0}\right]$ where $t_{0}$ is such that $(1-t) f(x)+t f(y) \in f(B)$ when $0 \leqq t \leqq t_{0}$. Proceeding as in the proof of Theorem 2, we have

$$
v(x, y, t)=x-t w(x, y)+o(t)
$$

so $\|v(x, y, t)\|$ is decreasing (as a function of $t$ ) when $\|v(x, y, t)\|>\|y\|$. By methods similar to those used in the proof of Theorem 2, we conclude that the set of allowable values of $t_{0} \in[0,1]$ is nonempty, open and closed. Hence we may choose $t_{0}=1$ and it follows that $f(B)$ is convex. 
THeOREM 6. Let $f: B \rightarrow Y$ be convex, $0 \neq x \in B$ and let $x^{\prime} \in T(x)$. Then the hyperplane $\left\{y \in Y: x^{\prime}\left[(D f(x))^{-1}(y)\right]=x^{\prime}\left[(D f(x))^{-1}(f(x))\right]\right\}$ is a supporting hyperplane for the convex set $f\left(B_{\|x\|}\right)$. If $y \neq 0, x^{\prime}(y)=0$ and $\|x+t y\|=\|x\|$ for $0<t<t_{0}$, then $x^{\prime}\left[(D f(x))^{-1}(f(x+t y))\right]=$ $x^{\prime}\left[(D f(x))^{-1}(f(x))\right]$ for $0<t<t_{0}$ (i.e., $f(x+t y)$ lies in the supporting hyperplane described above).

Proof. By Theorem 4, $\|y\|<\|x\|$ implies

$$
0<x^{\prime}(w(x, y))=x^{\prime}\left[(D f(x))^{-1}(f(x)-f(y))\right]
$$

so

$$
x^{\prime}\left[(D f(x))^{-1}(f(x))\right]>x^{\prime}\left[(D f(x))^{-1}(f(y))\right] .
$$

That is, all points in $f\left(B_{\|x\|}\right)$ lie on the same side of the hyperplane and the first part of the theorem is proved.

Expanding $f(x+t y)$ in a power series about $x$ we have

$$
f(x+t y)=f(x)+t D f(x)(y)+\frac{1}{2} t^{2} D^{2} f(x)(y, y)+o\left(t^{2}\right)
$$

so

$$
\begin{aligned}
w(x, x+t y) & =(D f(x))^{-1}(f(x)-f(x+t y)) \\
& =-t y-\frac{1}{2} t^{2}(D f(x))^{-1}\left(D^{2} f(x)(y, y)\right)+o\left(t^{2}\right)
\end{aligned}
$$

and

$$
x^{\prime}(w(x, x+t y))=-\frac{1}{2} t^{2} x^{\prime}(D f(x))^{-1}\left(D^{2} f(x)(y, y)\right)+o\left(t^{2}\right) .
$$

By Lemma 5 and Theorem 4, we conclude $x^{\prime}\left\{\lambda(D f(\lambda x))^{-1}\left(D^{2} f(\lambda x)(y\right.\right.$, $y))\} \leqq 0$ when $|\lambda| \leqq 1 /\|x\|$ with equality when $\lambda=0$. Since the function under consideration is a harmonic function of $\lambda$ we conclude $x^{\prime}\left((D f(x))^{-1}\left(D^{2} f(x)(y, y)\right)\right)=0$. Using induction on the higher order terms in the series we obtain the desired result, $x^{\prime}(w(x, x+t y))=0$.

Note that if $x$ is not an extreme point of the closed ball of radius $\|x\|, x \neq 0$, then $y$ and $x^{\prime}$ as given in the theorem do exist.

We now apply Theorem 6 to two particular spaces which show that the requirement that a holomorphic map of the unit ball be convex is very restrictive at least in some spaces. We asume that $(S, R, \mu)$ is a measure space with $\mu$ a positive measure defined on the $\sigma$-ring $R$ of subsets of $S$. We assume $S$ has two disjoint subsets of finite positive measure.

THEOREM 7. Let $X=L(\mu)$ (the space of complex valued integrable 
functions on $S)$. If $f: B \rightarrow Y$ is convex then $f(x)-f(0)$ is linear.

Proof. We may assume $Y=X, f(0)=0$ and $D f(0)=I$ (otherwise, replace $f$ by $\left.[D f(0)]^{-1}(f(x)-f(0))\right)$. Since $f(x)=x+1 / 2 \cdot D^{2} f(0)(x$, $x)+\cdots$, it is sufficient to show $D^{n} f(0)=0, n=2,3, \cdots$. The proof is by induction. Let $x=\alpha x_{E}+\beta x_{F}$ where $E$ and $F$ are disjoint sets of finite positive measure, $x_{E}$ and $x_{F}$ are characteristic functions for $E$ and $F$ respectively, $|\alpha| \mu(E)+|\beta| \mu(F)<1$ (so $x \in B$ ) and $\alpha \beta \neq 0$. Let $x^{\prime}$ be the real continuous linear functional on $X$ given by

$$
x^{\prime}(u)=\operatorname{Re}\left[|\alpha| / \alpha \int_{E} u d \mu+|\beta| / \beta \int_{F} u d \mu\right]
$$

and let

$$
y=-\alpha /(|\alpha| \mu(E))(1-i r) x_{E}+\beta /(|\beta| \mu(F))(1+i s) x_{F}
$$

where $r$ and $s$ are real.

Then $x^{\prime}(y)=0$ and

$$
\begin{aligned}
\| x & +t y \|=|\alpha|(\mu(E)-t /|\alpha|) \sqrt{1+(r t /(|\alpha| \mu(E)-t))^{2}} \\
& +|\beta|\left(\mu(F)+t /|\beta| \sqrt{1+(s t /(|\beta| \mu(F)+t))^{2}}\right. \\
= & \|x\|+|\alpha| \mu(E) r^{2} t^{2} /\left(2(|\alpha| \mu(E)-t)^{2}\right) \\
& +|\beta| \mu(F) s^{2} t^{2} /\left(2(|\beta| \mu(F)+t)^{2}\right)+o\left(t^{2}\right) \geqq\|x\|
\end{aligned}
$$

if $|t|$ is sufficiently small.

Therefore, using Theorem 4 and the definition of $Q$ we conclude $x^{\prime}(w(x+t y, x)) \geqq 0$ if $|t|$ is sufficiently small. Since $x^{\prime}(y)=0$, it follows as in the proof of Theorem 6 that $x^{\prime}(D t(x))^{-1}\left(D^{2} f(x)(y, y)\right)=0$.

Letting $L_{x}(\cdot, \cdot)=(D f(x))^{-1}\left(D^{2} f(x)(\cdot, \cdot)\right)$ we have

$$
\begin{aligned}
\operatorname{Re}\left[\int _ { E } \left(\alpha(1-i r)^{2} /\left(|\alpha| \mu^{2}(E)\right) L_{x}\left(x_{E}, x_{E}\right)\right.\right. \\
\quad-2 \beta(1-i r)(1+i s) /(|\beta| \mu(E) \mu(F)) \cdot \\
\left.\quad L_{x}\left(x_{E}, x_{F}\right)+|\alpha| \beta^{2}(1+i s)^{2} /\left(\alpha|\beta|^{2} \mu^{2}(F)\right) L_{x}\left(x_{F}, x_{F}\right)\right) d \mu \\
\quad+\int_{F}\left(\alpha^{2}|\beta|(1-i r)^{2} /\left(|\alpha|^{2} \beta \mu^{2}(E)\right) L_{x}\left(x_{E}, x_{E}\right)\right. \\
\quad-2 \alpha(1-i r)(1+i s) /(|\alpha| \mu(E) \mu(F)) \cdot \\
\left.\left.\quad L_{x}\left(x_{E}, x_{F}\right)+\beta(1+i s)^{2} /\left(|\beta| \mu^{2}(F)\right) L_{x}\left(x_{F}, x_{F}\right)\right) d \mu\right]=0 .
\end{aligned}
$$

In (4), if we replace $\alpha$ and $\beta$ by $\lambda \alpha$ and $\lambda \beta$ and multiply through by $|\lambda|$ we obtain an analytic function of $\lambda$ for $|\lambda|<R$ where $R>1$ which has 0 real part and is 0 at $\lambda=0$. Therefore the quantity in brackets is 0 . Since $\alpha, \beta, r$, and $s$ are variable, we conclude that each of the six terms above is 0 . Letting $\alpha$ and $\beta$ tend to 0 , we conclude 
that if $A$ and $B$ are disjoint measurable sets of finite measure then

$$
\int_{A} D^{2} f(0)\left(x_{A}, x_{A}\right) d \mu=\int_{B} D^{2} f(0)\left(x_{A}, x_{A}\right) d \mu=\int_{A} D^{2} f(0)\left(x_{A}, x_{B}\right) d \mu=0 .
$$

If $A$ and $E \cup F$ are disjoint and $A$ has finite positive measure, we may replace the functional $x^{\prime}$ in the argument above by $x_{1}^{\prime}$ where

$$
x_{1}^{\prime}(u)=x(u)+\operatorname{Re} \int_{A} \gamma u d \mu
$$

where $|\gamma| \leqq 1, \gamma$ complex. This leads to the conclusion, $\int_{A} D^{2} f(0)\left(x_{E}\right.$, $\left.x_{F}\right) d \mu=0$.

Hence we may now conclude that for simple functions $y$ we have $D^{2} f(0)(y, y)=0$. This implies $D^{2} f(0)(y, y)=0$ for all $y \in L(\mu)$. Similarly, $D^{k} f(0)(y, y, \cdots, y)=0$ for $k=3,4, \cdots$.

The theorem now follows from the power series for $f$ about 0 .

Choosing $S$ to be the positive integers and $\mu$ counting measure we obtain the following corollary.

Corollary 1. If $X=\ell^{1}$ and $f: B \rightarrow Y$ is convex then $f(x)-f(0)$ is linear.

THeOREM 8. Let $X=L^{\infty}(\mu)$ (the space of essentially bounded, complex valued, measurable functions on $S$ ) and suppose $f: B \rightarrow Y$ is convex. If $x, y \in B$ where $x(s)=y(s)$ for all $s \in E \subset S$ and $\mu(E)>0$ then $(D f(0))^{-1}(f(x))(s)=(D f(0))^{-1} f(y)(s)$ for almost all $s \in E$.

Proof. We may assume $Y=X, f(0)=0$ and $D f(0)=I$ (otherwise, replace $f$ by $\left.(D f(0))^{-1}(f(x)-f(0))\right)$. We will show that if $\mu(E)>0$, then for almost all $s \in E$ we have $f(x)(s)=f\left(x x_{E}\right)(s)$ (where $x_{E}$ is the characteristic function of $E$ ). We accomplish this by showing, $(D f(x))^{-1} D^{n} f(x)(y, \cdots, y)(s)=0$ a.e. on $E$ when $y(s)=0$ on $E(n \geqq 2)$ and $D^{2} f(x)\left(x_{E}, y\right)=0$ when $y=0$ on $E$. An induction argument will then show $D^{n} f(x)\left(x_{E}, y, y, \cdots, y\right)=\lim z \rightarrow 0\left[D^{n-1} f(x+z y)\left(x_{E}, y, y, \cdots, y\right)-\right.$ $\left.D^{n-1} f(x)\left(x_{E}, y, y, \cdots, y\right)\right] / z=0$ for $n \geqq 3$ when $y=0$ on $E$ and using the fact that $D^{n} f(x)$ is $n$-linear and symmetric (writing $y=y x_{E}+$ $\left.y\left(1-x_{E}\right)\right)$ we have

$$
\begin{aligned}
& D^{n} f(x)(y, y, \cdots, y)=D^{n} f(x)\left(y x_{E}, y x_{E}, \cdots, y x_{E}\right) \\
& \quad+D^{n} f(x)\left(y\left(1-x_{E}\right), y\left(1-x_{E}\right), \cdots, y\left(1-x_{E}\right)\right)
\end{aligned}
$$

for all $n \geqq 1$ when $E$ is measurable. Hence for $s \in E$ we have

$$
D^{n} f(0)(y, y, \cdots, y)(s)=D^{n} f(0)\left(y x_{E}, y x_{E}, \cdots, y x_{E}\right)(s) \text { a.e. on } E \text {. }
$$

Therefore, 


$$
\begin{aligned}
& f(x)(s)=\left[\sum_{k=1}^{\infty} \frac{1}{k !} D^{k} f(0)(x, x, \cdots, x)\right](s) \\
&=\left[\sum_{k=1}^{\infty} \frac{1}{k !} D^{k} f(0)(x, x, \cdots, x)(s)\right] \\
& \sum_{k=1}^{\infty} \frac{1}{k !} D^{k} f(0)\left(x x_{E}, x x_{E}, \cdots, x x_{E}\right)(s) \\
&=f\left(x x_{E}\right)(s) \text { for almost all } s \in E .
\end{aligned}
$$

Let $E$ be a set of positive measure, let $0<\varepsilon<r<1$ and

$$
x=\alpha x_{E}+\left(1-x_{E}\right) u, u \in X
$$

where $|\alpha|=r$ and $\|u\| \leqq r-\varepsilon$.

Let $y \in B$ satisfy

$$
y(s)=0 \text { if } s \in E .
$$

Then $\|x+\beta y\|=\|x\|=r$ for $|\beta|$ sufficiently small. Let $x^{\prime}$ be the real continuous linear functional on $X$ given by

$$
x^{\prime}(v)=\operatorname{Re} \int_{E}\left(\frac{\bar{\alpha}}{r \mu(E)}\right) v d \mu .
$$

Then $x^{\prime} \in T(x)$ and $x^{\prime}(\beta y)=0$ so Theorem 6 applies. Since $\arg \beta$ is arbitrary we may therefore conclude

$$
(D f(x))^{-1} D^{2} f(x)(y, y)(s)=0 \text { a.e. on } E \text {. }
$$

But $(D f(x))^{-1} D^{2} f(x)(y, y)$ is an analytic function of $\alpha$ for $|\alpha|<1(E, u$, and $y$ remain fixed) which satisfies (9) when $|\alpha|>\|u\|$. Hence (9) holds when $|\alpha|<1$ and $\|u\|<1$. For arbitrary $x \in B$, we may approximate $x x_{E}$ on $E$ by simple functions to see that (9) holds for all $x \in B$ if $y(s)=0$ on $E$. An induction argument shows that (9) holds when $D^{2} f(x)(y, y)$ is replaced by $D^{n} f(x)(y, y, \cdots, y)$ where $n \geqq$ 2 and $y=0$ on $E$.

We now wish to show $D^{2} f(x)\left(x_{E}, y\right)=0$ for all $x \in B$ and $y \in X$ satisfying $y(s)=0$ if $s \in E$.

Let $x$ and $y$ be given by (7) and (8) and set $h=\beta y+t \alpha x_{E}$ where $|\beta|=t^{1 / 2}$. Then for $t$ sufficiently small, $\|x+h\| \sqrt{1+t^{2}}\|x\|$ and

where

$$
\begin{aligned}
w\left(x, \frac{1}{\sqrt{1+t^{2}}}(x+h)\right) & =(D f(x))^{-1}\left(f(x)-f\left(\frac{1}{\sqrt{1+t^{2}}}\right)(x+h)\right) \\
& =-v-(D f(x))^{-1}\left(D^{2} f(x)(v, v)\right)-\cdots
\end{aligned}
$$

$$
v=\left(\frac{1}{\sqrt{1+t^{2}}}-1\right) x+\frac{1}{\sqrt{1+t^{2}}} \cdot h \text {. }
$$

With $x^{\prime}$ as above, applying Theorem 4 we obtain 


$$
\begin{aligned}
0 \leqq & x^{\prime}\left(w\left(x, \frac{1}{\sqrt{1+t^{2}}}(x+h)\right)\right) \\
= & \operatorname{Re}\left(\frac{-i r t \beta}{\mu(E)} \int_{E}(D f(x))^{-1} D^{2} f(x)\left(x_{E}, y\right) d \mu\right) \\
& +o\left(t^{2}\right) .
\end{aligned}
$$

Since $|\beta|=t^{1 / 2}$ and $\arg \beta$ is arbitrary, we conclude

$$
\int_{E}(D f(x))^{-1} D^{2} f(x)\left(x_{E}, y\right) d \mu=0 \text { when } y=0 \text { on } E .
$$

Let $F \subset S, F$ measurable, and $\mu(F)>0$. Equation (10) implies

$$
\int_{F \cap E}(D f(x))^{-1} D^{2} f(x)\left(x_{E}, y\right) d \mu=0 \text {. }
$$

Also,

$$
\begin{aligned}
\int_{F-E} & L_{x}\left(x_{E}, y\right) d \mu=\int_{F-E}\left[L_{x}\left(y x_{F}, x_{E}\right)\right. \\
& +\frac{1}{2} L_{x}\left(x_{E}+y\left(1-x_{F}\right), x_{E}+y\left(1-x_{F}\right)\right)-\frac{1}{2} L_{x}\left(x_{E}, x_{E}\right) \\
& \left.-\frac{1}{2} L_{x}\left(y\left(1-x_{F}\right), y\left(1-x_{F}\right)\right)\right] d \mu=0
\end{aligned}
$$

for the first term is 0 by (10) applied to $F-E$ and the other 3 terms are 0 by (9) applied to $F-E$. Hence we conclude $(D f(x))^{-1} D^{2} f(x)\left(x_{E}\right.$, $y)(s)=0$ a.e. on $S$ so $D^{2} f(x)\left(x_{E}, y\right)=0$.

Again taking $S$ to be the positive integers with $\mu$ the counting measure we obtain the following corollary.

Corollary 2. If $X=\ell^{\infty}$ and $f: B \rightarrow Y$ is convex then $f(x)-$ $f(0)=D f(0)(g(x))$ where $g(x)=\left(g_{1}\left(x_{1}\right), g_{2}\left(x_{2}\right), \cdots\right)$ and $g_{k}\left(x_{k}\right)=x_{k}+$ $a_{2 k} x_{k}^{2}+\cdots$ maps $\left|x_{k}\right|<1$ onto a convex domain.

4. Discussion. The techniques used in this paper may also be used to extend the concepts of close-to-convexity and spirallikeness to Banach spaces. There are at least two different ways of extending the analytic condition for close-to-convexity to Banach spaces and these apparently lead to different classes of functions. Results concerning these concepts are as yet incomplete and will be discussed in a later paper.

\section{REFERENCES}

1. L. A. Harris, Schwarz's Lemma in normed linear spaces, Proc. Natl. Acad. Sci. U.S.A., 64 (4), (1969), 1014-1017. 
2. E. Hille and R. S. Phillips, Functional Analysis and Semigroups, Amer. Math. Soc. Colloq. Publ., 31 (1957).

3. Takeshi Matsuno, Star-like theorems and convex-like theorems in the complex vector space, Sci. Rep. Toko, Kyoiku Daigaku, Sect A 5 (1955), 88-95.

4. M. S. Robertson, Applications of the subordination principle to univalent functions, Pacific J. Math., 11 (1961), 315-324.

5. G. T. Schwartz, Nonlinear Functional Analysis, Courant Inst. of Math. Sciences NYU, 1965.

6. T. J. Suffridge, The principle of subordination applied to functions of several variables, Pacific J. Math., 33 (1970), 241-248.

Received March 27, 1972.

UNIVERSITY OF KENTUCKY 



\section{PACIFIC JOURNAL OF MATHEMATICS}

\section{EDITORS}

D. Gilbarg and J. Milgram

Stanford University

Stanford, California 94305

\author{
R. A. Beaumont \\ University of Washington \\ Seattle, Washington 98105
}

J. DuGUNDJI

Department of Mathematics

University of Southern California

Los Angeles, California 90007

Richard ARENS

University of California

Los Angeles, California 90024

\section{ASSOCIATE EDITORS}
E. F. BECKENBACH
B. H. NeumanN
F. WOLF
K. YosHIDA

\section{SUPPORTING INSTITUTIONS}

UNIVERSITY OF BRITISH COLUMBIA

CALIFORNIA INSTITUTE OF TECHNOLOGY

UNIVERSITY OF CALIFORNIA

MONTANA STATE UNIVERSITY

UNIVERSITY OF NEVADA

NEW MEXICO STATE UNIVERSITY

OREGON STATE UNIVERSITY

UNIVERSITY OF OREGON

OSAKA UNIVERSITY

\author{
UNIVERSITY OF SOUTHERN CALIFORNIA \\ STANFORD UNIVERSITY \\ UNIVERSITY OF TOKYO \\ UNIVERSITY OF UTAH \\ WASHINGTON STATE UNIVERSITY \\ UNIVERSITY OF WASHINGTON
* * * *
AMERICAN MATHEMATICAL SOCIETY \\ NAVAL WEAPONS CENTER
}

The Supporting Institutions listed above contribute to the cost of publication of this Journal, but they are not owners or publishers and have no responsibility for its content or policies.

Mathematical papers intended for publication in the Pacific Journal of Mathematics should be in typed form or offset-reproduced, (not dittoed), double spaced with large margins. Underline Greek letters in red, German in green, and script in blue. The first paragraph or two must be capable of being used separately as a synopsis of the entire paper. The editorial "we" must not be used in the synopsis, and items of the bibliography should not be cited there unless absolutely necessary, in which case they must be identified by author and Journal, rather than by item number. Manuscripts, in duplicate if possible, may be sent to any one of the four editors. Please classify according to the scheme of Math. Rev. Index to Vol. 39. All other communications to the editors should be addressed to the managing editor, Richard Arens, University of California, Los Angeles, California, 90024.

50 reprints are provided free for each article; additional copies may be obtained at cost in multiples of 50 .

The Pacific Journal of Mathematics is issued monthly as of January 1966. Regular subscription rate: $\$ 48.00$ a year (6 Vols., 12 issues). Special rate: $\$ 24.00$ a year to individual members of supporting institutions.

Subscriptions, orders for back numbers, and changes of address should be sent to Pacific Journal of Mathematics, 103 Highland Boulevard, Berkeley, California, 94708.

PUBLISHED BY PACIFIC JOURNAL OF MATHEMATICS, A NON-PROFIT CORPORATION

Printed at Kokusai Bunken Insatsusha (International Academic Printing Co., Ltd.), 270, 3-chome Totsuka-cho, Shinjuku-ku, Tokyo 160, Japan. 


\section{Pacific Journal of Mathematics}

\section{Vol. 46, No. 2 December, 1973}

Christopher Allday, Rational Whitehead products and a spectral sequence of

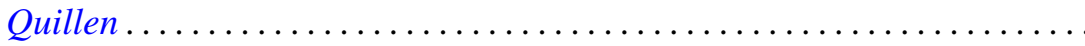

James Edward Arnold, Jr., Attaching Hurewicz fibrations with fiber

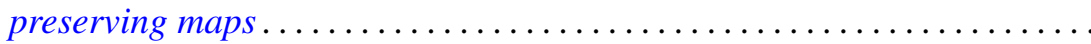

Catherine Bandle and Moshe Marcus, Radial averaging transformations with various metrics.................................

David Wilmot Barnette, A proof of the lower bound conjecture for convex

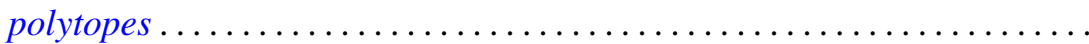

Louis Harvey Blake, Simple extensions of measures and the preservation of

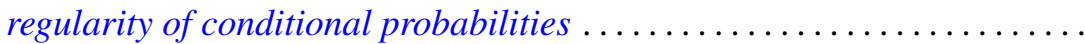

James W. Cannon, New proofs of Bing's approximation theorems for

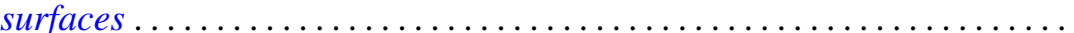

C. D. Feustel and Robert John Gregorac, On realizing HNN groups in

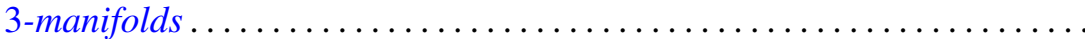

Theodore William Gamelin, Iversen's theorem and fiber algebras . . . . . . . . 389

Daniel H. Gottlieb, The total space of universal fibrations . . . . . . . . . . . .

Yoshimitsu Hasegawa, Integrability theorems for power series expansions of

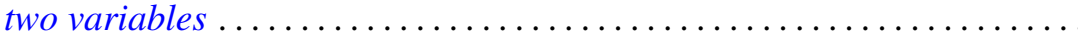

Dean Robert Hickerson, Length of period simple continued fraction expansion of $\sqrt{ } d$

Herbert Meyer Kamowitz, The spectra of endomorphisms of the disc algebra.

Dong S. Kim, Boundedly holomorphic convex domains

Daniel Ralph Lewis, Integral operators on $\mathscr{L}_{p}$-spaces ...

John Eldon Mack, Fields of topological spaces . . . . . . . . .

V. B. Moscatelli, On a problem of completion in bornology

Ellen Elizabeth Reed, Proximity convergence structures. .

Ronald C. Rosier, Dual spaces of certain vector sequence spaces .

Robert A. Rubin, Absolutely torsion-free rings

Leo Sario and Cecilia Wang, Radial quasiharmonic functions . .

James Henry Schmerl, Peano models with many generic classes .

H. J. Schmidt, The $\mathscr{F}$-depth of an $\mathscr{F}$-projector ............

Edward Silverman, Strong quasi-convexity. . . . . . . . . . . . . . . . . 549

Barry Simon, Uniform crossnorms ....................... 555

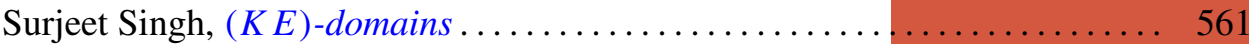

Ted Joe Suffridge, Starlike and convex maps in Banach spaces . . . . . . . . 575

Milton Don Ulmer, $C$-embedded $\Sigma$-spaces . . . . . . . . . . . . . . . . 591

Wolmer Vasconcelos, Conductor, projectivity and injectivity . . . . . . . . . 603 